\title{
A New Dual Circularly Polarized Feed Employing a Dielectric Cylinder-Loaded Circular Waveguide Open End Fed by Crossed Dipoles
}

\author{
Jae-Hoon Bang, ${ }^{1}$ Soon-Woo Choi, ${ }^{2}$ Jae-Woo Noh, ${ }^{2}$ Joo-Young Lim, ${ }^{2}$ Dong-Hyun Kim, \\ Dae-Oh Kim, ${ }^{3}$ and Bierng-Chearl $\mathrm{Ahn}^{2}$ \\ ${ }^{1}$ Duta Technology Co. Ltd., Daejeon, Republic of Korea \\ ${ }^{2}$ Department of Radio and Communications Engineering, Chungbuk National University, Cheongju, Republic of Korea \\ ${ }^{3}$ Naro Space Center at the Korea Aerospace Research Institute, Goheung-gun, Chonnam, Republic of Korea \\ Correspondence should be addressed to Bierng-Chearl Ahn; bician@cbu.ac.kr
}

Received 22 July 2016; Revised 23 October 2016; Accepted 9 November 2016

Academic Editor: Xianming Qing

Copyright (c) 2016 Jae-Hoon Bang et al. This is an open access article distributed under the Creative Commons Attribution License, which permits unrestricted use, distribution, and reproduction in any medium, provided the original work is properly cited.

This paper presents a new dual circularly polarized feed that provides good axial ratio over wide angles and low cross-polarized radiation in backward direction. A circular waveguide open end is fed with two orthogonally polarized waves in phase quadrature by a pair of printed crossed dipoles and a compact connectorized quadrature hybrid coupler. The waveguide aperture is loaded with a dielectric cylinder to reduce the cross-polarization beyond 90 degrees off the boresight. The fabricated feed has, at $5.5 \mathrm{GHz}$, 6.33- $\mathrm{dBic}$ copolarized gain, 3- $\mathrm{dB}$ beamwidth of $106^{\circ}, 10$ - $\mathrm{dB}$ beamwidth of $195^{\circ}, 3$ - $\mathrm{dB}$ axial ratio beamwidth of $215^{\circ}$, maximum crosspolarized gain of $-21.4 \mathrm{dBic}$, and $27-\mathrm{dB}$ port isolation. The reflection coefficient of the feed is less than $-10 \mathrm{~dB}$ at $4.99-6.09 \mathrm{GHz}$.

\section{Introduction}

High-gain dual circularly polarized reflector antennas are employed in such applications as aeronautical telemetry, meteorological radar, ground penetrating radar, and satellite communication. Dual circular polarization offers mitigation against multipath fading and MIMO capability for wireless communication. Crucial to the reflector performance are the feed characteristics. The feed can be configured either for a prime-focus reflector with the feed gain of around 6$12 \mathrm{~dB}$ or for a dual reflector antenna with the feed gain of around $15-25 \mathrm{~dB}$. In this paper, we present a new dual circularly polarized feed suitable for high-performance primefocus reflector applications. The proposed antenna employs a dielectric cylinder-loaded circular waveguide open end excited by crossed dipoles for wide axial ratio beamwidth and low cross-polarization in backward direction.

Various methods of realizing a low gain $(6-12 \mathrm{~dB})$ antenna with dual linear or dual circular polarization have been established which include crossed dipoles $[1,2]$, dual-fed patches [3], waveguide apertures [4], crossed slots [5], and dielectric resonators [6].

High-performance prime-focus reflector antenna feeds are often realized using circular waveguides [7]. A dual circularly polarized wave can be generated inside a circular waveguide by exciting two orthogonally polarized fields having a 90-degree phase difference. To excite dual polarized waves in a circular or square waveguide, various structures have been employed such as the orthomode transducer [8], the septum polarizer [9], quadruple ridges [10], the shorted ring patch [11], and dual coaxial probes [12]. The quadrature phase can be realized by using either a waveguide polarizer [13] or a quadrature hybrid coupler.

Crossed dipoles have widely been employed for GNSS reception [14], mobile phone base station [1,2], and feeding a square horn [15], a cavity [16], and a cup [17]. The distinction between a cavity and a cup is somewhat arbitrary. The former is used for the large aperture. A cup can also be called an open-ended circular waveguide. 


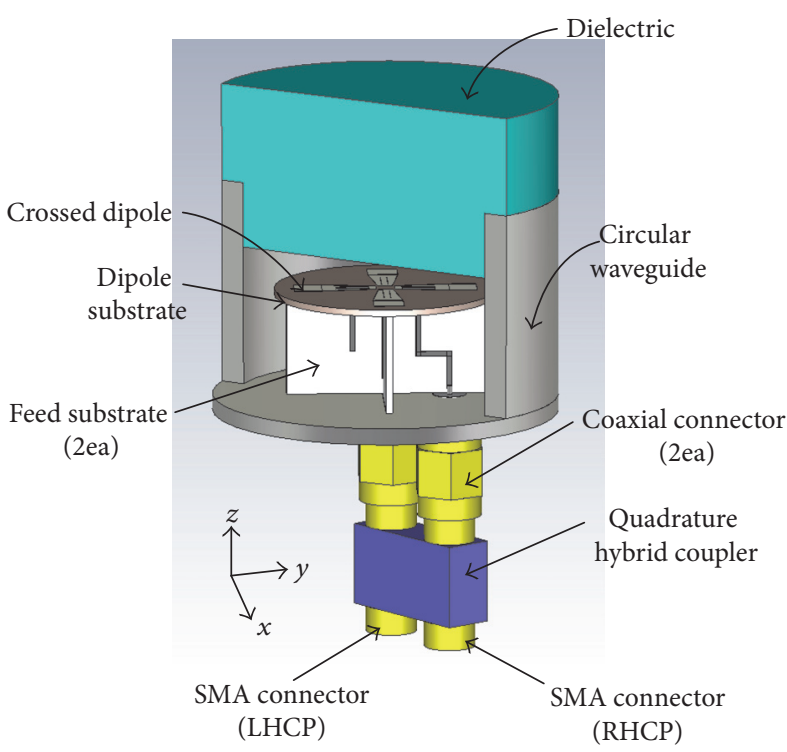

(a)

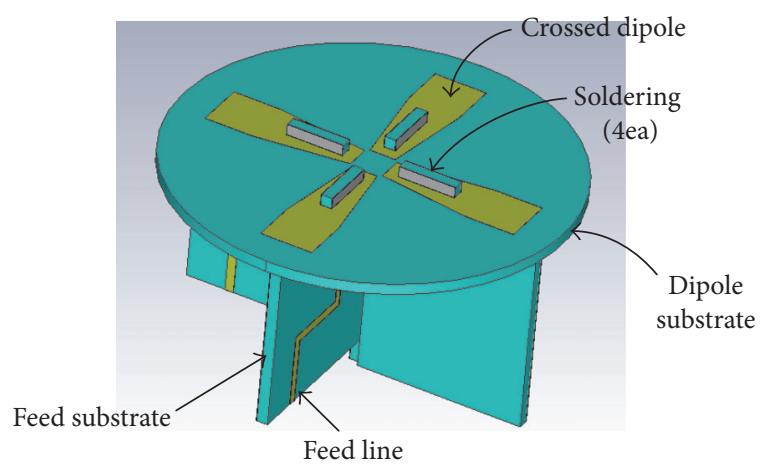

(b)

FIGURE 1: Structure of the proposed feed. (a) Overall structure and (b) crossed dipoles with associated feed circuits.

Crossed dipoles can be realized on the same plane as the feed circuit [1] or on a separate plane on top of the feed circuit [2]. Loading a waveguide aperture with dielectric material has been employed for impedance matching [18] or for radiation pattern improvements [19].

Antennas with wider axial ratio beamwidth have been proposed by many authors [20-24]. Mak and Luk employed a pair of crossed shorted bowtie patches and a crossed dipole pair fed by a Wilkinson power divider with a 90-degree phase delay line [20]. Park and Lee proposed a narrow-band circularly polarized antenna that employs mu-negative transmission line [21]. Shi and coworkers proposed an antenna design that employs a disk-loaded monopole, a microstrip curl radiator, and tilted parasitic wires [22]. Wang and coworkers employed two interlaced coplanar dipoles backed by a rectangular cavity [23]. Wei and coworkers proposed a ring probe-fed metallic cavity antenna [24]. Existing designs for antennas with wide axial ratio beamwidth vary in their complexities and many of them cannot be employed as a feed without adding attachment fixture and radome, which might require design adjustment or redesign. Some of them operate only as a single circularly polarized radiator.

In this paper, we propose a new rugged dual circularly polarized antenna suitable for high-performance primefocus reflector antenna feed. The proposed antenna can be employed as a real-world feed without any modification or design adjustment since its structure is such that it can be exposed in the weather without difficulty.

To realize a new feed, we have employed a circular waveguide open end excited by a pair of crossed dipoles. A dielectric cylinder is placed at the open end aperture for cross-polarization reduction at angles beyond $90^{\circ}$ from the antenna axis. The $90^{\circ}$ phase difference is realized using a compact commercial quadrature hybrid coupler directly connected to the crossed dipoles. The design and measurement of the proposed feed are presented below.

\section{Antenna Design}

Figure 1(a) shows the structure of the proposed dual circularly polarized feed. The feed consists of two printed crossed dipoles exciting a circular waveguide loaded with a dielectric cylinder on its aperture, and a quadrature hybrid coupler connected to the crossed dipoles via coaxial connectors. The crossed dipole pair are closely matched in their characteristics and fed in phase quadrature. Crossed dipoles on a finite ground plane show low axial ratio only within small angles around the beam axis. To increase the axial ratio beamwidth, crossed dipoles are placed inside a circular waveguide of proper diameter. In this case, the feed radiation characteristics are predominantly determined by the waveguide open end aperture.

Circular waveguide open ends with radius of 0.4 to 0.6 times the wavelength provide good $E$ - and $H$-plane beam pattern symmetry and thus low cross-polarization for angles up to 90 degrees from the waveguide axis [7]. When they are used as a circular polarized radiator, they offer a low axial ratio over the same angular range. In the proposed feed, a crossed dipole pair is utilized to launch two orthogonal polarizations with high polarization isolation in a circular waveguide. A high-performance quadrature hybrid coupler is employed to excite the crossed dipole with both right- and left-hand polarizations. The result is a dual polarized feed having a low axial ratio over wide angles.

Figure 1(b) shows the structure of the crossed dipoles. The dipoles are fed by a microstrip line with an integrated balun whose detailed geometry is shown in Figures 3(b)-3(d). The Taconic RF-35 substrate with dielectric constant of 3.5, 


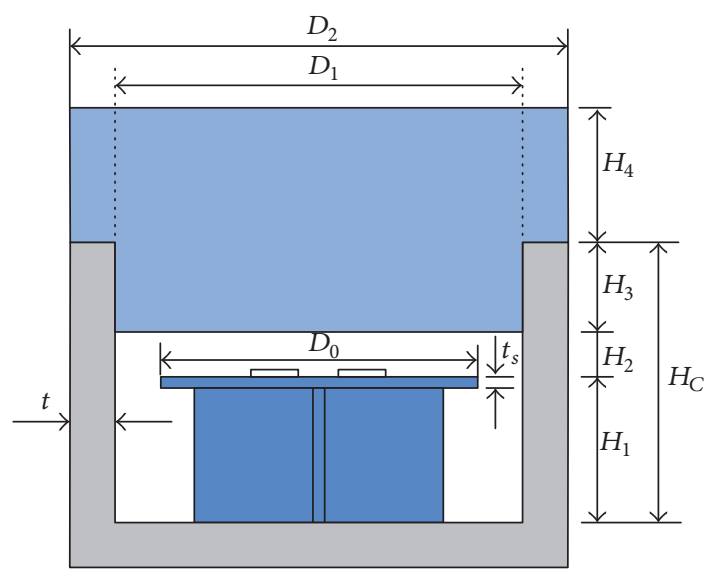

Figure 2: Design parameters of the proposed feed.

loss tangent of 0.0018 , and thickness of $0.76 \mathrm{~mm}$ have been employed. The dipole can be placed on the same plane as the feeding microstrip line [1]. Simulations have shown that lower back radiation is obtained when the dipole is orthogonal to feed circuit surface.

Wide-angle cross-polarization is effectively suppressed by the dielectric cylinder of proper height placed on the waveguide aperture. The polycarbonate with dielectric constant of 2.78 and loss tangent of 0.0005 has been employed.

Figures 2 and 3 show the dimensional parameters of the proposed feed. The design of the feed starts with initial values of major dimensions: circular waveguide diameter $D_{1}=0.6 \lambda$ for low back radiation and for low cross-polarization [7], dipole height $H_{1}=0.25 \lambda$, dipole length $2\left(D_{L 1}+D_{L 2}\right)+s=$ $0.45 \lambda$, aperture distance $H_{C}=0.5 \lambda$, and dielectric cylinder height $H_{4}=0.25 \lambda$. The dipole-to-dielectric cylinder gap $\mathrm{H}_{2}$ is set to a suitable minimum value $(0.08 \lambda)$ to preserve the dipole's impedance bandwidth.

Design parameters are successively adjusted for low axial ratio over wide angles and for low back radiation. The dipole length and dipole feed network dimensions are adjusted for good impedance matching over the operating frequency range. The distance between the dipole feeding ports is made exactly the same as the spacing between two coaxial ports of the quadrature hybrid coupler so that a direct mating is possible.

The circular waveguide diameter is optimum when it is the initial value $(0.6 \lambda)$ as described in [7]. The feed performance is not sensitive to the dipole height $H_{1}$ as far as it is around $0.25 \lambda$. The dipole-to-waveguide aperture distance does not significantly affect the feed performance so that it is set to $0.2 \lambda$. The dielectric cylinder height $H_{4}$ is crucial in reducing cross-polarization at wide angles and an optimum value is obtained by parameter sweep. The CST Microwave Studio $^{\mathrm{TM}}$ is employed in the design of the proposed feed.

Dimensions of the optimally designed feed are as follows with units in mm: $D_{0}=27.00, D_{1}=36.00, D_{2}=42.00, t=3.0$, $t_{s}=0.76, H_{0}=12.25, H_{1}=13.00, H_{2}=4.75, H_{3}=8.00, H_{4}=$ $12.00, H_{C}=25.00, H_{s}=1.50, D_{L 1}=6.58, D_{L 2}=3.35, D_{W 1}=$ $1.80, D_{W 2}=3.65, s=2.28, L=24.00, w=0.6, a_{1}=3.97, a_{2}=$
3.73, $a_{3}=8.00, a_{4}=8.30, a_{5}=5.10, a_{6}=6.00, a_{7}=1.15, a_{8}=$ $5.85, b_{1}=3.97, b_{2}=4.23, b_{3}=7.00, b_{4}=8.20, b_{5}=5.10, b_{6}=5.00$, $b_{7}=2.15, b_{8}=6.35, W_{b}=2.0, W_{s}=4.00, D_{b}=10.27$.

Design results shown in Figures 4(a) and 4(b) illustrate the main concepts of the proposed feed. Curves marked with " $x$ " are for the gain of cross-polarized radiation. Figure 4(a) shows that the cross-polarization levels at angles beyond $90^{\circ}$ from the antenna boresight are significantly reduced by using the dielectric cylinder. The narrow axial ratio beamwidth of the crossed dipoles on a ground plane is greatly increased by placing the crossed dipoles inside an open-ended circular waveguide. As shown in Figure 4(b), the 3-dB axial ratio beamwidth is about $52^{\circ}$ when the cross dipoles are placed on a finite ground plane while it is broadened to $175^{\circ}$ with the use of the open-ended circular waveguide.

Figure 5 shows the RHCP gain and phase patterns of the designed feed at frequencies 5.0, 5.5, and 6.0 GHz. The LHCP patterns of the feed are almost identical to the RHCP patterns. The feed's phase center is at a point $9.2 \mathrm{~mm}$ away from the dielectric cylinder's top surface toward the crossed dipoles. The $10-\mathrm{dB}$ beamwidth of the designed feed is $150^{\circ}-163^{\circ}$ at 5-6 GHz. Within 10- $\mathrm{dB}$ beamwidth angle, the feed's phase variation is $9-12^{\circ}$ at $5-6 \mathrm{GHz}$. The simulated reflection and isolation performances of the proposed feed are presented with measurements.

\section{Fabrication and Measurements}

The designed feed has been fabricated and tested. The photograph of the fabricated antenna is shown in Figure 6. The crossed dipoles and the associated feed circuits have been fabricated using standard PCB manufacturing processes. The feed circuit boards have been tightly placed in their respective grooves on the waveguide back short, which is fixed to the waveguide wall using four screws.

A coax-to-microstrip transition with a two-hole panel mount flange and an SMA male connector has been used to connect the microstrip feed line of each dipole to a small commercial quadrature hybrid coupler (Cernex Wave CHC0408U318T).

The quadrature hybrid coupler has dimensions of $25.7 \times$ $13 \times 11 \mathrm{~mm}^{3}$ and its measured performance at $5-6 \mathrm{GHz}$ is as follows: reflection at both input ports less than $-28 \mathrm{~dB}$, isolation between two ports greater than $30 \mathrm{~dB}$, phase balance better than $\pm 0.7^{\circ}$, and magnitude balance better than $\pm 0.35 \mathrm{~dB}$. The quadrature hybrid coupler is directly connected to the coax-to-microstrip transition without using cables.

Figure 7 shows the reflection coefficient and isolation of the fabricated antenna measured using HP $8720 \mathrm{C}$ network analyzer. The reflection coefficient is less than $-10 \mathrm{~dB}$ at $4.99-$ $6.20 \mathrm{GHz}$ at port 1 (RHCP) and at $4.94-6.09 \mathrm{GHz}$ at port 2 (LHCP). The port-to-port isolation is greater than $27 \mathrm{~dB}$ over 5-6 GHz.

Figure 8 shows the co- and cross-polarization gain patterns and the axial ratio patterns of the fabricated antenna at $5.5 \mathrm{GHz}$ measured in an anechoic chamber far-field range. The axial ratio has been measured by using a transmitting antenna with dual linear polarizations and the proposed 


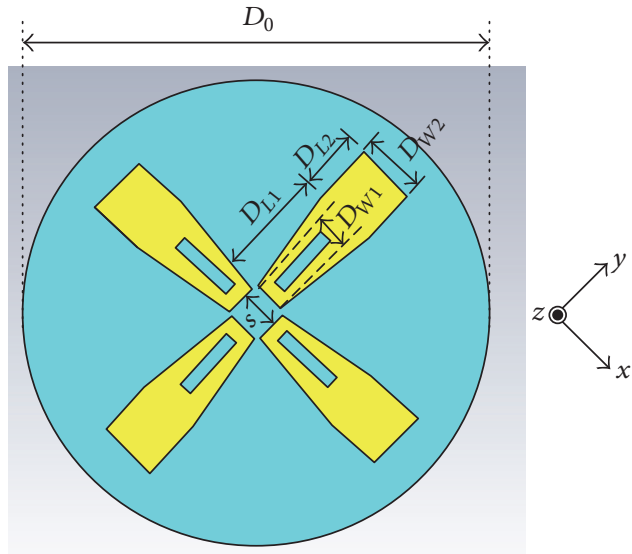

(a)

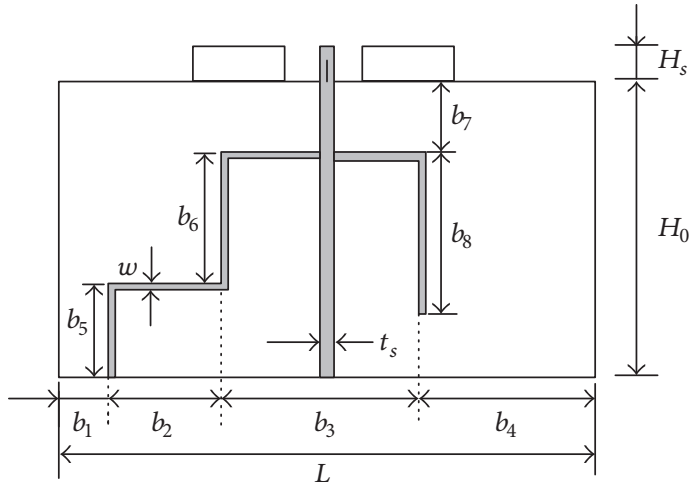

(c)

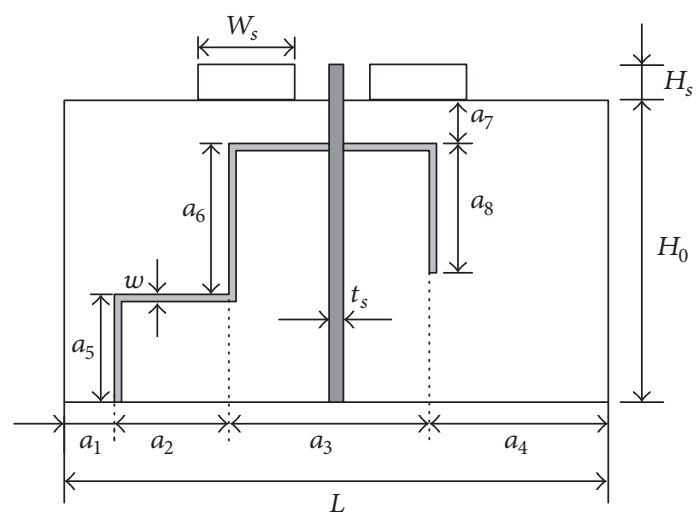

(b)

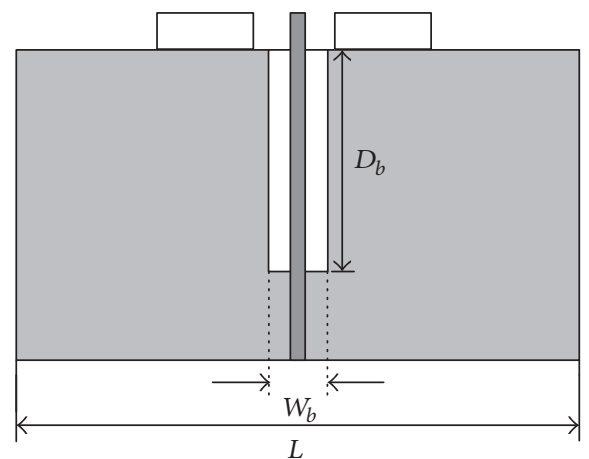

(d)

FIGURE 3: Design parameters of the crossed dipoles. (a) Crossed dipoles, (b) the dipole feed circuits for RHCP, (c) the dipole feed circuits for LHCP, and (d) the backside of the feed circuits for RHCP and LHCP.

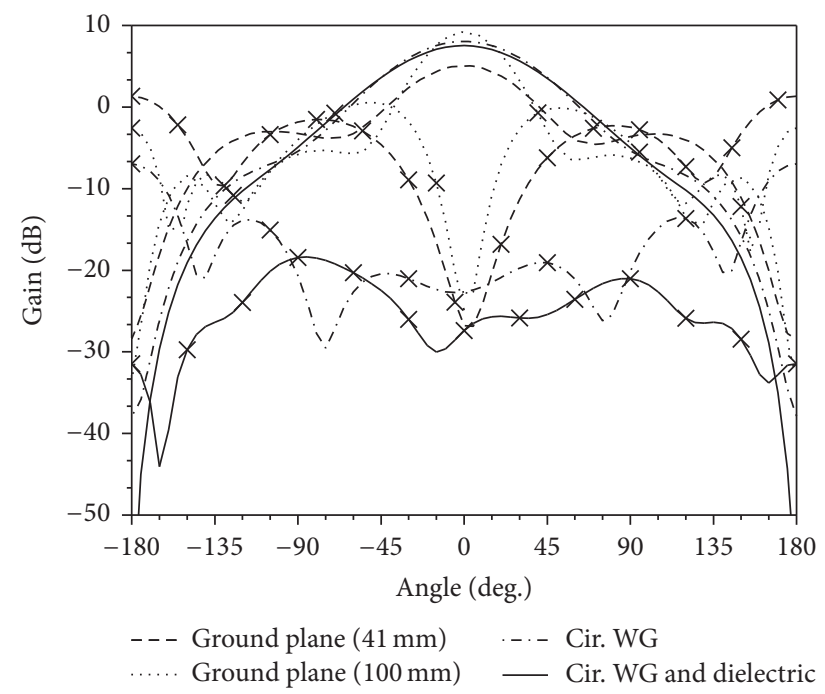

(a)

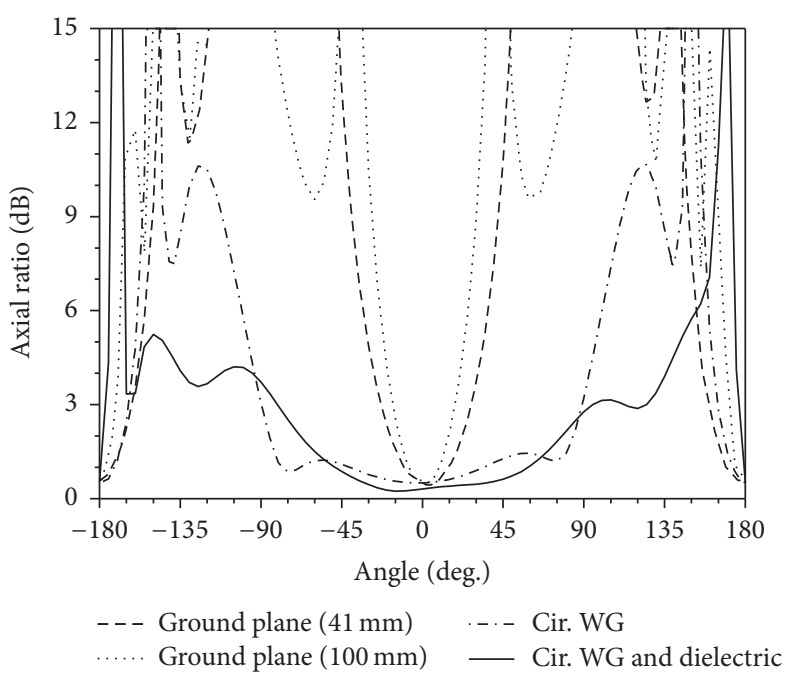

(b)

FIGURE 4: (a) Gain and (b) axial ratio patterns of the crossed dipoles of various configurations on $z x$-plane. 


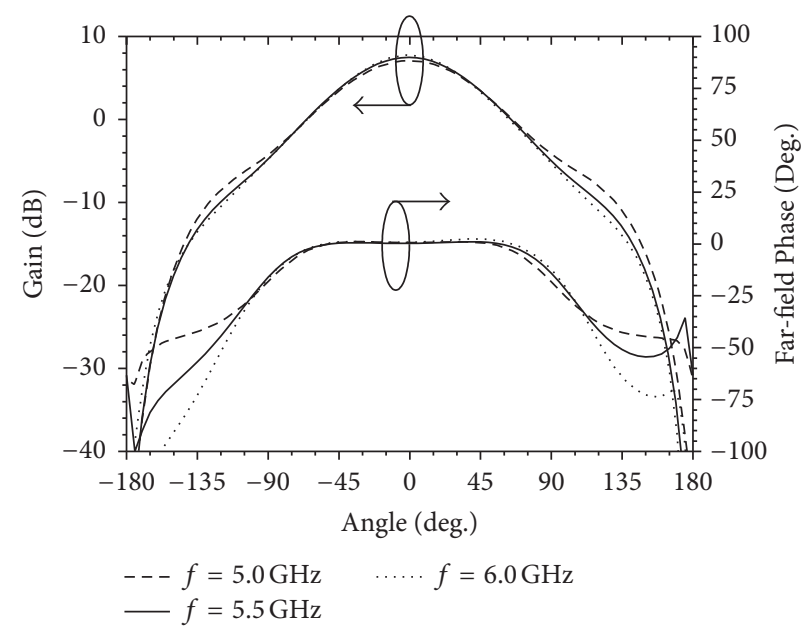

FIGURE 5: RHCP gain and phase patterns of the proposed feed on $z x$-plane.

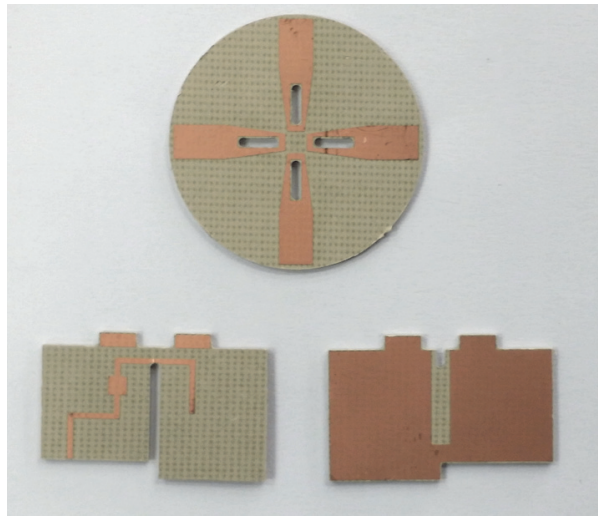

(a)

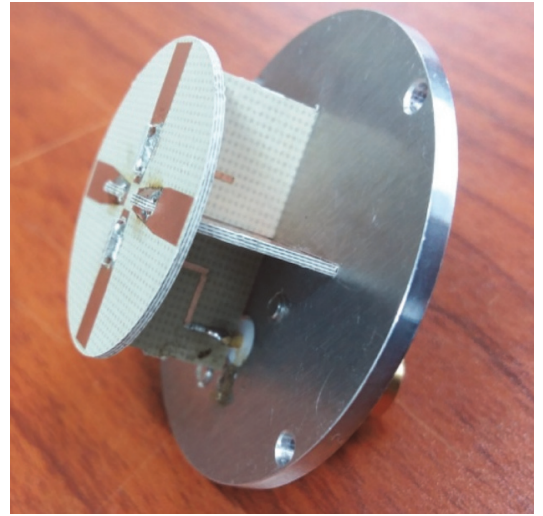

(b)

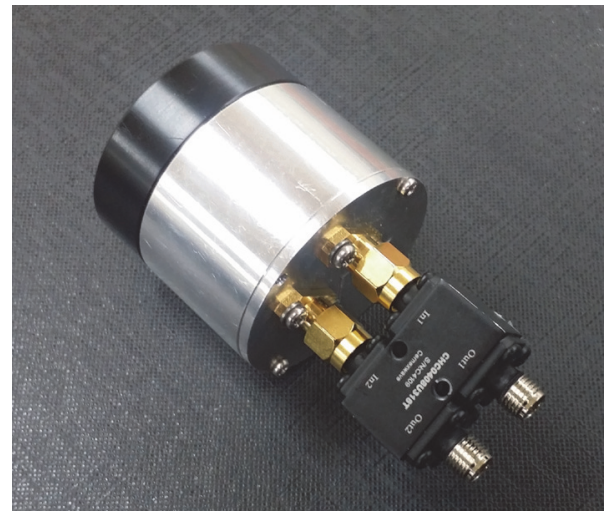

(c)

FIgURE 6: Fabricated feed. (a) Crossed dipoles and feed circuits, (b) crossed dipoles installed on the back short, and (c) the assembled antenna.

antenna as a receiving antenna. Magnitude and phase of the received signal of each polarization are recorded as the antenna under test is rotated. The axial ratio is calculated from the measured data.

The fabricated feed has the following characteristics at $5.5 \mathrm{GHz}$ : maximum gain of $6.33 \mathrm{dBic}, 3-\mathrm{dB}$ beamwidth of $106^{\circ}, 10-\mathrm{dB}$ beamwidth of $195^{\circ}, 3-\mathrm{dB}$ axial ratio beamwidth of $215^{\circ}$, and maximum cross-polarization of $-21.4 \mathrm{dBic}$. The fabricated feed has low back radiation. Figure 9 shows the gain and axial ratio versus the frequency. Measured gain ranges from $6.1 \mathrm{dBic}$ to $6.7 \mathrm{dBic}$ and measured axial ratio ranges from $0.4 \mathrm{~dB}$ to $1.4 \mathrm{~dB}$ over $5-6 \mathrm{GHz}$. In Figures 7-9, measurements agree reasonably well with the simulation. 


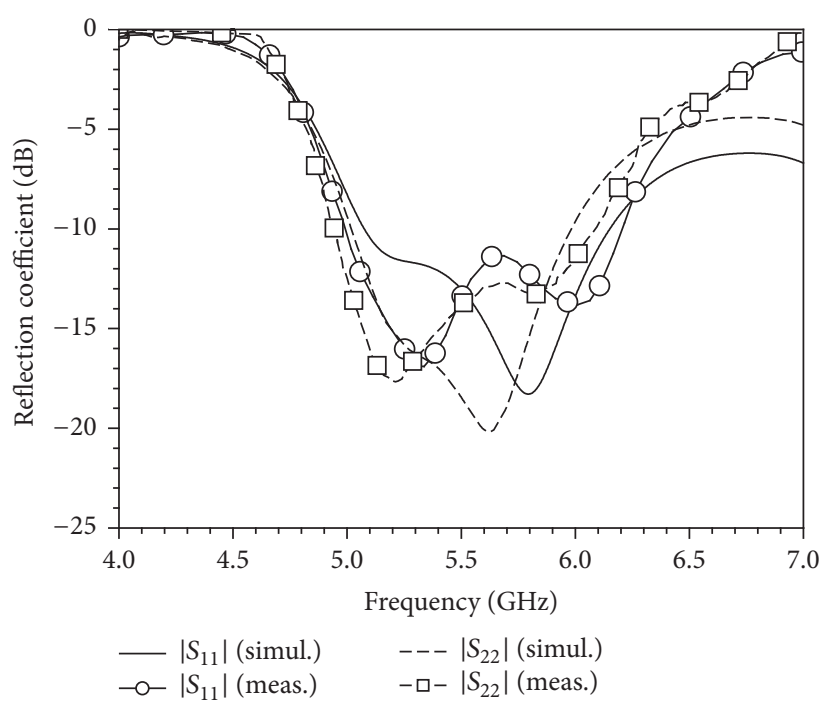

(a)

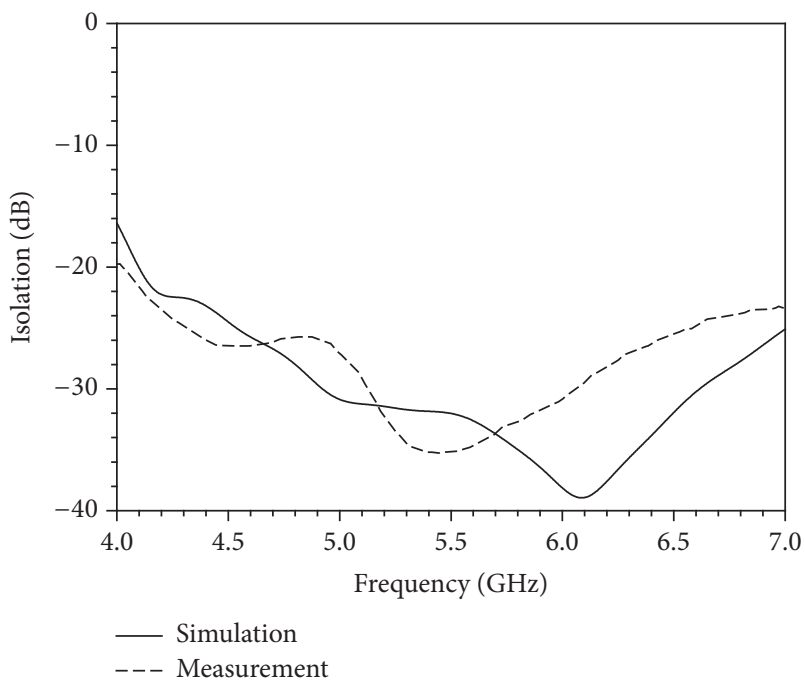

(b)

Figure 7: (a) Reflection and (b) isolation performance of the fabricated feed.

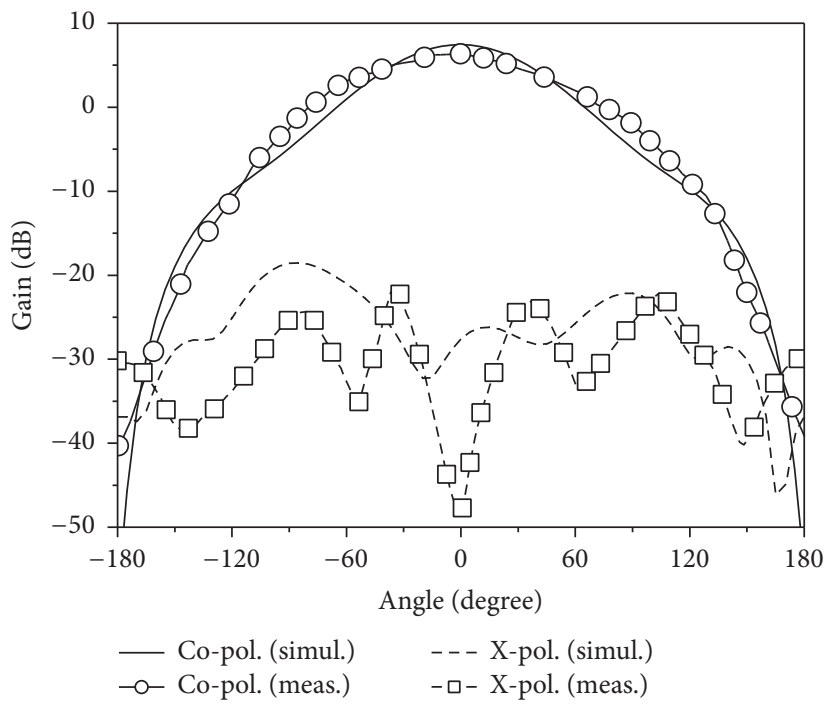

(a)

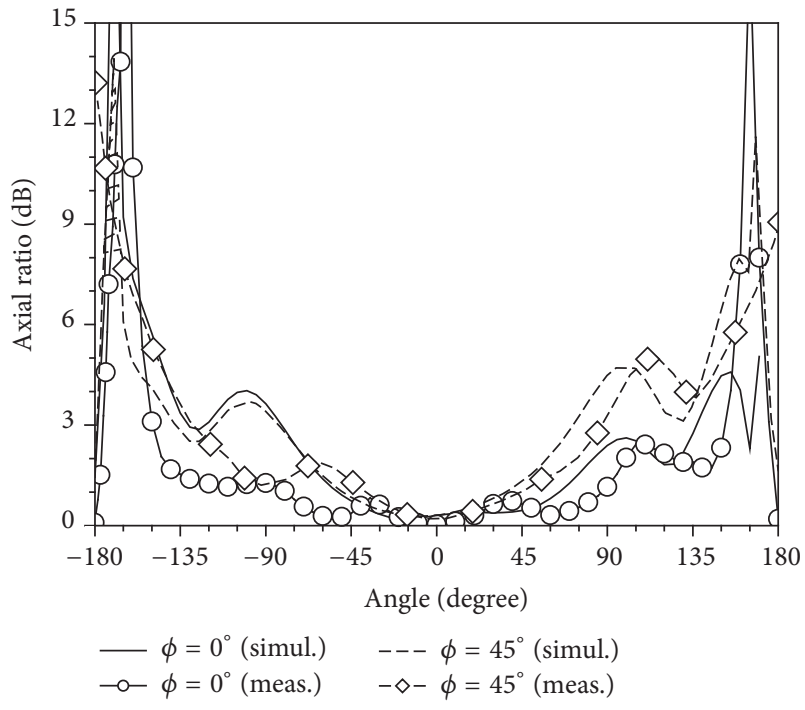

(b)

FIgURE 8: (a) Co-/cross-polarized gain patterns and (b) axial ratio patterns of the fabricated feed at $5.5 \mathrm{GHz}$.

\section{Conclusion}

A new dual circularly polarized feed is presented and demonstrated for wide beam radiations. The antenna employs a dielectric cylinder-loaded circular waveguide open end fed by a pair of printed crossed dipoles. A $90^{\circ}$ phase difference for circular polarization is provided with a compact quadrature hybrid coupler by Cernex Wave Inc. Starting from initial dimensions, an optimum design has been obtained. The designed feed has been fabricated and tested. Measurements have shown that the proposed feed has such desirable characteristics for use in prime-focus reflector antennas as low axial ratio and low cross-polarization over wide angles, low back radiation, circular symmetric gain pattern, flat phase pattern, and high polarization isolation over its operating bandwidth. The proposed feed has 6.3-dBic copolarized gain, 3 - $\mathrm{dB}$ beamwidth of $106^{\circ}, 10$ - $\mathrm{dB}$ beamwidth of $195^{\circ}, 3-\mathrm{dB}$ axial ratio beamwidth of $215^{\circ}$, maximum crosspolarized gain of $-21.4 \mathrm{dBic}$, and $27-\mathrm{dB}$ port isolation. The proposed antenna can be used as a feed for high-performance prime-focus reflector antennas having dual circular polarization.

\section{Competing Interests}

The authors declare that there is no conflict of interests regarding the publication of this paper. 


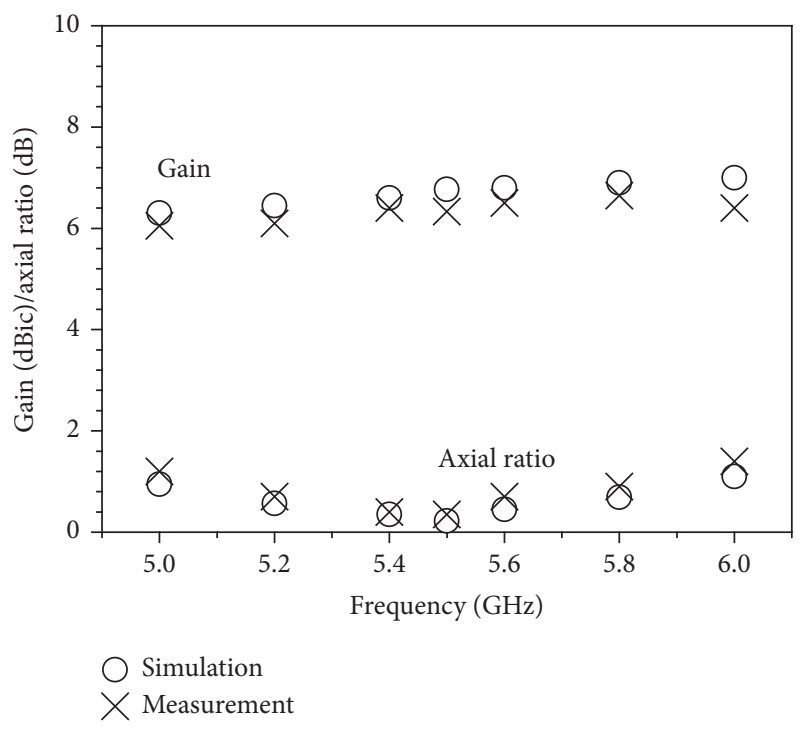

FIGURE 9: Gain and axial ratio of the fabricated feed versus the frequency.

\section{References}

[1] S.-Y. Eom, I.-P. Hong, and J.-M. Kim, "Broadband printed cross-dipole element with four polarization reconfigurations for mobile base station array antenna applications," International Journal of Antennas and Propagation, vol. 2011, Article ID 427015, 10 pages, 2011.

[2] S.-L. Zuo, Q.-Q. Liu, and Z.-Y. Zhang, "Wideband dualpolarized crossed-dipole antenna with parasitical crossed-strip for base station applications," Progress in Electromagnetics Research C, vol. 48, pp. 159-166, 2014.

[3] Y. Cheng, Y. Li, and W. Lu, "A novel compact dual-polarized antenna," International Journal of Antennas and Propagation, vol. 2016, Article ID 6304356, 5 pages, 2016.

[4] J.-Y. Lim, J. Nyambayar, J.-Y. Yun et al., "High-performance dual-circularly polarized reflector antenna feed," ETRI Journal, vol. 36, no. 6, pp. 889-893, 2014.

[5] R. K. Saini and S. Dwari, "A broadband dual circularly polarized square slot antenna," IEEE Transactions on Antennas and Propagation, vol. 64, no. 1, pp. 290-294, 2016.

[6] W. C. Wong and K. W. Leung, "Circularly polarized dielectric resonator antenna excited by dual conformal strips of unequal lengths," Microwave and Optical Technology Letters, vol. 29, no. 5, pp. 348-350, 2001.

[7] A. D. Olver, P. J. B. Clarricoats, A. A. Kishk, and L. Shafai, in Microwave Horns and Feeds, pp. 185-199, IEEE Press, New York, NY, USA, 1994.

[8] J. Uher, J. Bornemann, and U. Rosenberg, Waveguide Components for Antenna Feed Systems: Theory and CAD, Artech House, Norwood, Mass, USA, 1993.

[9] G. H. Schennum and T. M. Skiver, "Antenna feed element for low circular cross-polarization," in Proceedings of the IEEE Aerospace Conference, pp. 135-150, Snowmass at Aspen, Colo, USA, February 1997.

[10] A. Raghunathan, N. U. Shankar, and N. V. G. Sarma, "Multi octave prime focus feeds for a parabolic reflector," in Proceedings of the 28th URSI General Assembly, New Delhi, India, October 2005.
[11] Z. A. Pour and L. Shafai, "A novel dual-mode dual-polarized circular waveguide feed excited by concentrically shorted ring patches," IEEE Transactions on Antennas and Propagation, vol. 61, no. 10, pp. 4917-4925, 2013.

[12] J. B. West and L. J. Gatewood, "High-isolation broadband polarization diverse circular waveguide feed," U.S. Pat. 6,452,561 B1, Septmber 2002.

[13] S. Y. Eom and Y. B. Korchemkin, "A new comb circular polarizer suitable for millimeter-band application," ETRI Journal, vol. 28, no. 5, pp. 656-659, 2008.

[14] H. H. Tran, S. X. Ta, and I. Park, "Single-feed, wideband, circularly polarized, crossed bowtie dipole antenna for global navigation satellite systems," The Korean Institute of Electromagnetic Engineering \& Science, vol. 14, pp. 299-305, 2014.

[15] A. Kumar, "Crossed-dipole fed square horn," International Journal of Electronics, vol. 64, no. 6, pp. 955-960, 1988.

[16] T. Milligan, Modern Antenna Design, Wiley/IEEE Press, New York, NY, USA, 2nd edition, 2005.

[17] A. R. Mahnad, "Broadband cup antennas," U.S. Pat. 4,668,956 A, May 1998.

[18] J. J. Lee and R.-S. Chu, "Aperture matching of a dielectric loaded circular waveguide element array," IEEE Transactions on Antennas and Propagation, vol. 37, no. 3, pp. 395-399, 1989.

[19] A. D. Olver, P. J. B. Clarricoats, A. A. Kishk, and L. Shafai, in Microwave Horns and Feeds, pp. 392-401, IEEE Press, New York, NY, USA, 1994.

[20] K. M. Mak and K. M. Luk, "A circularly polarized antenna with wide axial ratio beamwidth," IEEE Transactions on Antennas and Propagation, vol. 57, no. 10, pp. 3309-3312, 2009.

[21] B. Park and J. Lee, "Compact circularly polarized antenna with wide 3-dB axial-ratio beamwidth," IEEE Antennas and Wireless Propagation Letters, vol. 15, pp. 410-413, 2016.

[22] L. Shi, H. Sun, and X. Lu, "A composite antenna with wide circularly polarized beamwidth," Microwave and Optical Technology Letters, vol. 51, no. 10, pp. 2461-2464, 2009.

[23] H. Wang, X. Wang, S. F. Liu, P. Li, and X. W. Shi, "Broadband circularly polarized antenna with high gain and wide axial ratio beamwidth," Microwave and Optical Technology Letters, vol. 57, no. 2, pp. 377-381, 2015.

[24] K. Wei, Z. Zhang, Y. Zhao, and Z. Feng, "Design of a ring probe-fed metallic cavity antenna for satellite applications," IEEE Transactions on Antennas and Propagation, vol. 61, no. 9, pp. 4836-4839, 2013. 


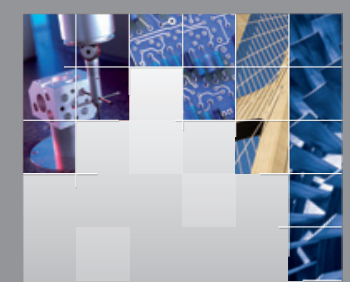

\section{Enfincering}
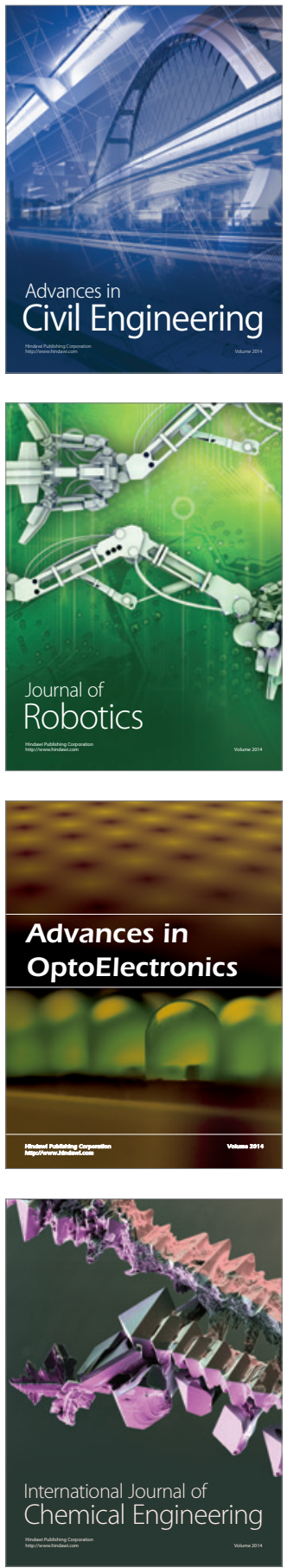

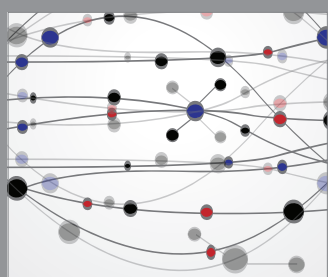

The Scientific World Journal

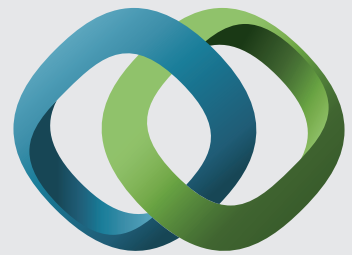

\section{Hindawi}

Submit your manuscripts at

http://www.hindawi.com
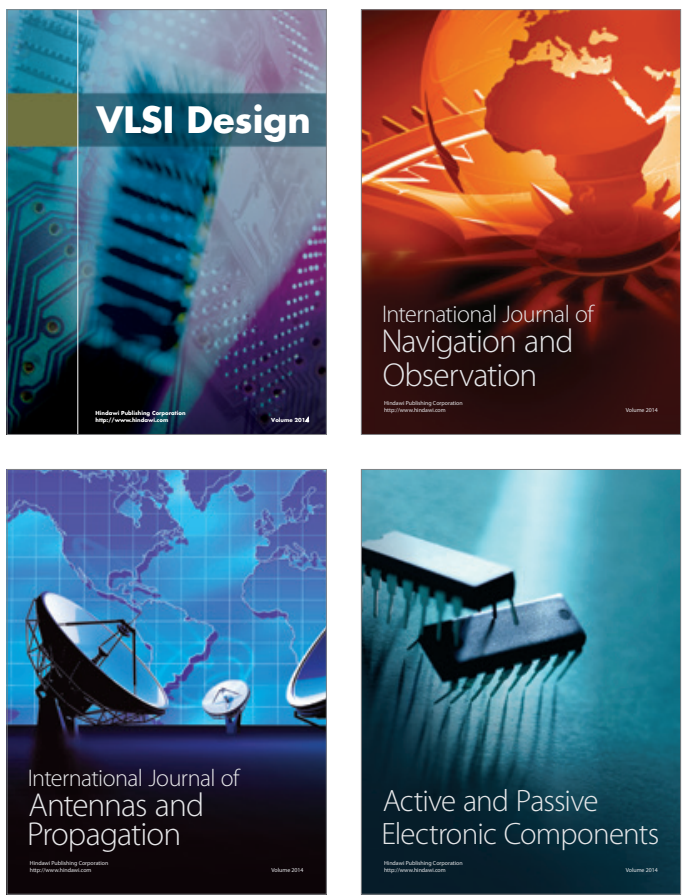
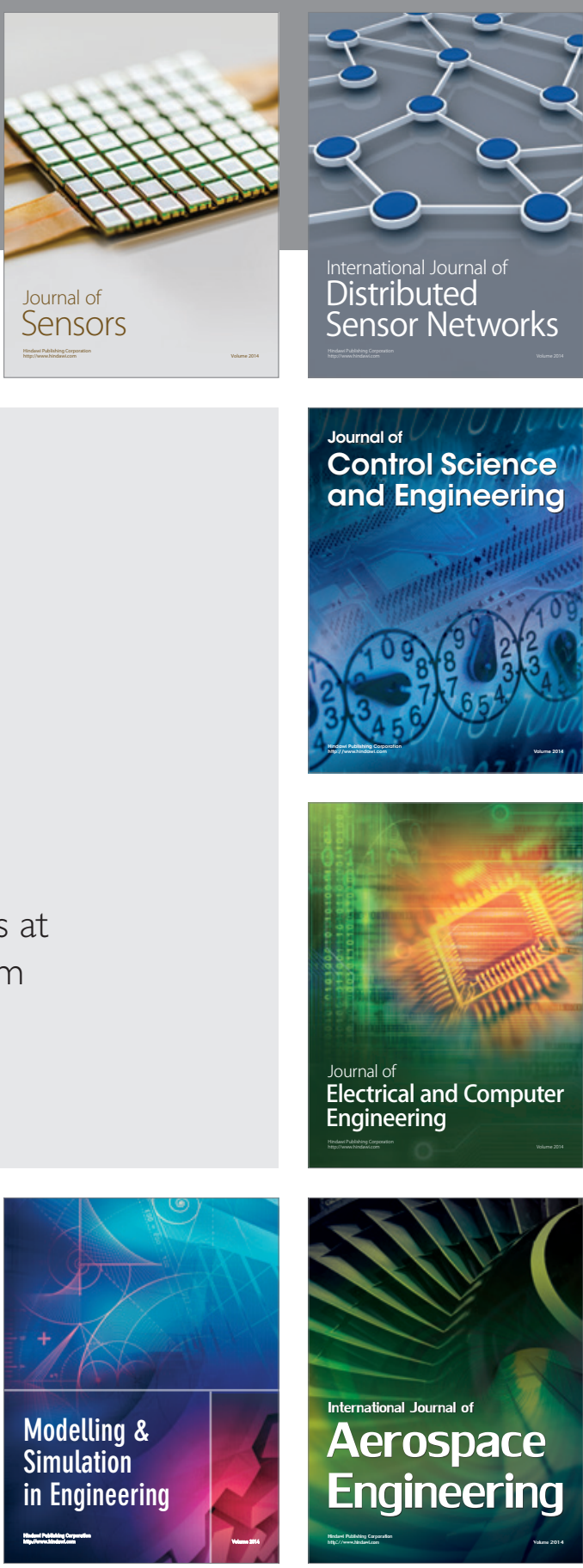

International Journal of

Distributed

Sensor Networks

Journal of

Control Science

and Engineering
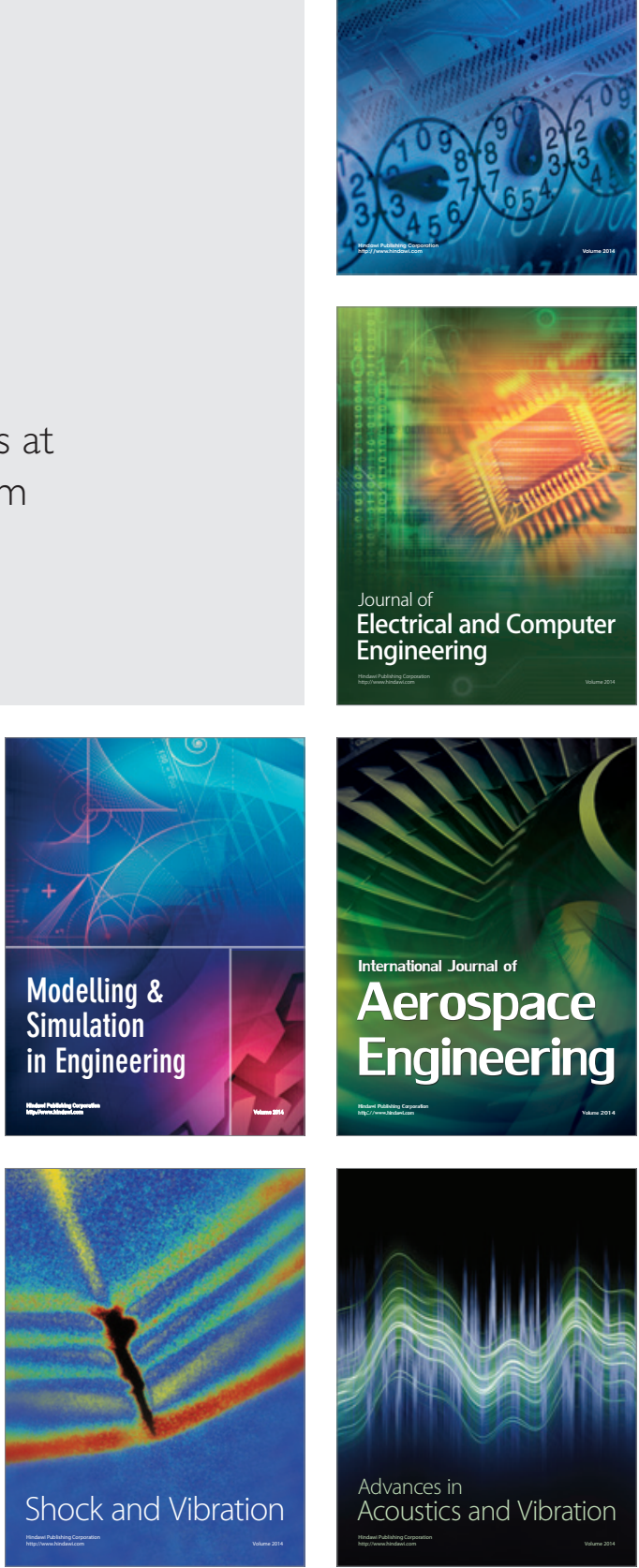